\title{
Paradoxical herniation after decompressive craniectomy provoked by lumbar puncture or ventriculoperitoneal shunting
}

\author{
Claire J. Creutzfeldt, MD, ${ }^{1}$ Marcelo D. Vilela, MD,, ${ }^{2,3}$ and William T. Longstreth Jr., MD ${ }^{1,4}$ \\ Departments of ${ }^{1}$ Neurology, ${ }^{2}$ Neurological Surgery, and ${ }^{4}$ Epidemiology, University of Washington, Seattle, Washington; and \\ ${ }^{3}$ Mater Dei Hospital, Belo Horizonte, Minas Gerais, Brazil
}

\begin{abstract}
OBJECT Two patients who underwent decompressive craniectomy after head trauma deteriorated secondary to paradoxical herniation, one after lumbar puncture and the other after ventriculoperitoneal shunting. They motivated the authors to investigate further provoked paradoxical herniation.
\end{abstract}

METHODS The authors reviewed the records of 205 patients who were treated at a single hospital with decompressive craniectomy for head trauma to identify those who had had lumbar puncture performed or a ventriculoperitoneal shunt placed after craniectomy but before cranioplasty. Among the patients who met these criteria, those with provoked paradoxical herniation were identified. The authors also sought to identify similar cases from the literature. Exact binomials were used to calculate $95 \%$ Cls.

RESULTS None of 26 patients who underwent a lumbar puncture within 1 month of craniectomy deteriorated, whereas 2 of 10 who underwent a lumbar puncture 1 month afterward did so $(20 \%$ [ $95 \% \mathrm{Cl} 2.4 \%-55.6 \%])$. Similarly, after ventriculoperitoneal shunting, 3 of 10 patients deteriorated $(30 \%[95 \% \mathrm{Cl} 6.7 \%-65.2 \%])$. Timing of the procedure and the appearance of the skin flap were important factors in deterioration after lumbar puncture but not after ventriculoperitoneal shunting. A review of the literature identified 15 additional patients with paradoxical herniation provoked by lumbar puncture and 7 by ventriculoperitoneal shunting.

CONCLUSIONS Lumbar puncture and ventriculoperitoneal shunting carry substantial risk when performed in a patient after decompressive craniectomy and before cranioplasty. When the condition that prompts decompression (such as brain swelling associated with stroke or trauma) requires time to resolve, risk is associated with lumbar puncture performed $\geq 1$ month after decompressive craniectomy.

http://thejns.org/doi/abs/10.3171/2014.11.JNS141810

KEY WORDS craniectomy; spinal puncture; ventriculoperitoneal shunt; herniation; cranioplasty; trauma

$\mathrm{T}$ HE term "syndrome of the trephined" was coined in the 1930s to describe a variety of symptoms in patients with cranial defects that were relieved by cranioplasty "in a large majority of cases." drome of the sinking skin flap" was applied to a subset of patients with large concave cranial defects after decompressive craniectomy who experienced spontaneous neurological deterioration, some of whom showed improvement after cranioplasty. ${ }^{22}$ In more recent series, both retrospective and prospective, improvement in neurological function after cranioplasty was reported in $16 \%-26 \% .^{7,18}$ We cared for 2 patients with posttraumatic decompressive craniectomy whose marked deterioration caused by so-called paradoxi- cal herniation ${ }^{16}$ was not spontaneous but provoked, one by a lumbar puncture and the other by a ventriculoperitoneal shunt. These 2 patients prompted us to review our experience and those in the literature.

\section{Methods}

We describe here both index cases, to whom we refer as Patients A and B to ensure anonymity. We also investigated a series of consecutive patients at a single hospital who underwent decompressive craniectomy with dural augmentation for traumatic brain injury over a specific time interval to identify those who also underwent a lum- 
bar puncture or ventriculoperitoneal shunting between the time of the decompressive craniectomy and cranioplasty, death, or loss to follow-up. We documented those whose condition deteriorated after the procedure to gain some understanding of the frequency of this complication and of the factors related to the deterioration. Finally, we reviewed the literature for similar cases by searching the phrases quoted in the introduction in PubMed and the bibliographies of all papers so identified.

\section{Results \\ Patient A: Index Case of Deterioration After Lumbar Puncture}

This patient was struck by a car while riding his bicycle without a helmet. He sustained skull fractures, a right-sided subdural hematoma, and a right frontal contusion for which a right-sided decompressive craniectomy was performed soon after admission. The patient was discharged to a rehabilitation facility approximately 1 month after the accident. When readmitted for a cranioplasty approximately 3 months after the accident, the patient was noted to be alert and oriented, and he had residual weakness and pain on the left side. The skin flap was sunken on the right. The planned procedure was cancelled because of a urinary tract infection. A cranioplasty was performed at a later admission, but the bone became infected and had to be removed. The patient continued to improve neurologically and participated in inpatient rehabilitation. Approximately 1 year after the initial injury, the patient returned to the hospital with a fever and elevated white blood cell count. A lumbar puncture was performed as part of the fever evaluation; meningitis was not found. The patient's condition subsequently deteriorated, and he became comatose over the ensuing 24 hours. Serial imaging revealed paradoxical herniation (Fig. 1). Despite treatment with fluids, positioning with his head down, and a blood patch, the patient did not improve neurologically to the level of functioning before the lumbar puncture. When last seen approximately 2 months after the lumbar puncture, the patient was minimally responsive but able to follow some simple commands on the right side.

\section{Patient B: Index Case of Deterioration After Ventriculoperitoneal Shunting}

This patient fell from a skateboard without a helmet and sustained skull fractures, a left-sided subdural hematoma, and bilateral frontal contusions for which a left-sided decompressive craniectomy was performed soon after admission. Initially, the patient was recovering well but then worsened after approximately 2 weeks. The patient was found to have hydrocephalus, and a right frontal ventriculoperitoneal shunt with an adjustable pressure valve was placed. After intensive inpatient rehabilitation, the patient was discharged home approximately 6 weeks after the injury. Between 2 and 3 months after the accident, the patient began to experience increasing right-sided weakness and word-finding problems. The skin flap was sunken on the left. These problems then worsened, and imaging revealed paradoxical herniation (Fig. 2). The patient's weakness and aphasia improved after adjustment of

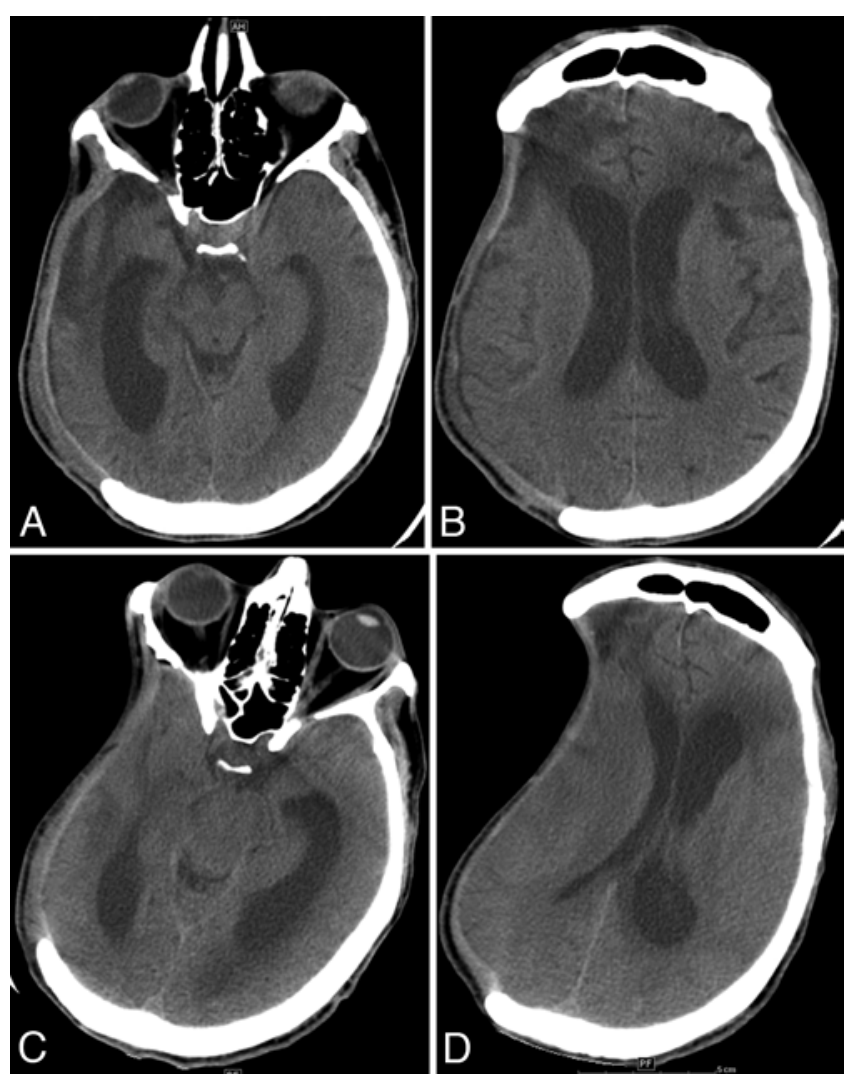

FIG. 1. Patient A. Axial head CT images obtained 1 day before (A and B) and 1 day after (C and D) lumbar puncture, showing paradoxical herniation provoked by lumbar puncture.

the shunt pressure valve to reduce drainage and eventually disappeared after a cranioplasty, which was performed approximately 5 months after the accident.

\section{Case Series at Harborview Medical Center}

After securing approval from the University of Washington Human Subjects Committee, we used administrative databases to identify each patient who underwent decompressive craniectomy for a traumatic brain injury at Harborview Medical Center in Seattle, Washington, before November 1, 2006. As part of the procedure, the dura is always opened with a stellate-type incision to permit brain herniation through the craniectomy defect. The medical records of 205 patients were screened to find documentation that spinal fluid was assayed or that a ventriculoperitoneal shunt was placed after decompressive craniectomy and before cranioplasty. Details about the lumbar puncture were often lacking. All analyses were conducted using Stata version 13.1 (StataCorp).

The initial case in the series was from July 15, 1991. The index case for the lumbar puncture was included among the group of 205 patients, whereas the index case for shunting was not included because treatment occurred after 2006. The screen yielded 36 patients who underwent lumbar puncture and 10 patients who underwent ventriculoperitoneal shunting who met these criteria. Deterioration that resulted from paradoxical herniation occurred in 2 of the 36 patients after lumbar puncture $(5.6 \%$ [exact binomial $95 \% \mathrm{CI} 0.7 \%-18.7 \%]$ ) and in 3 of the 10 patients 

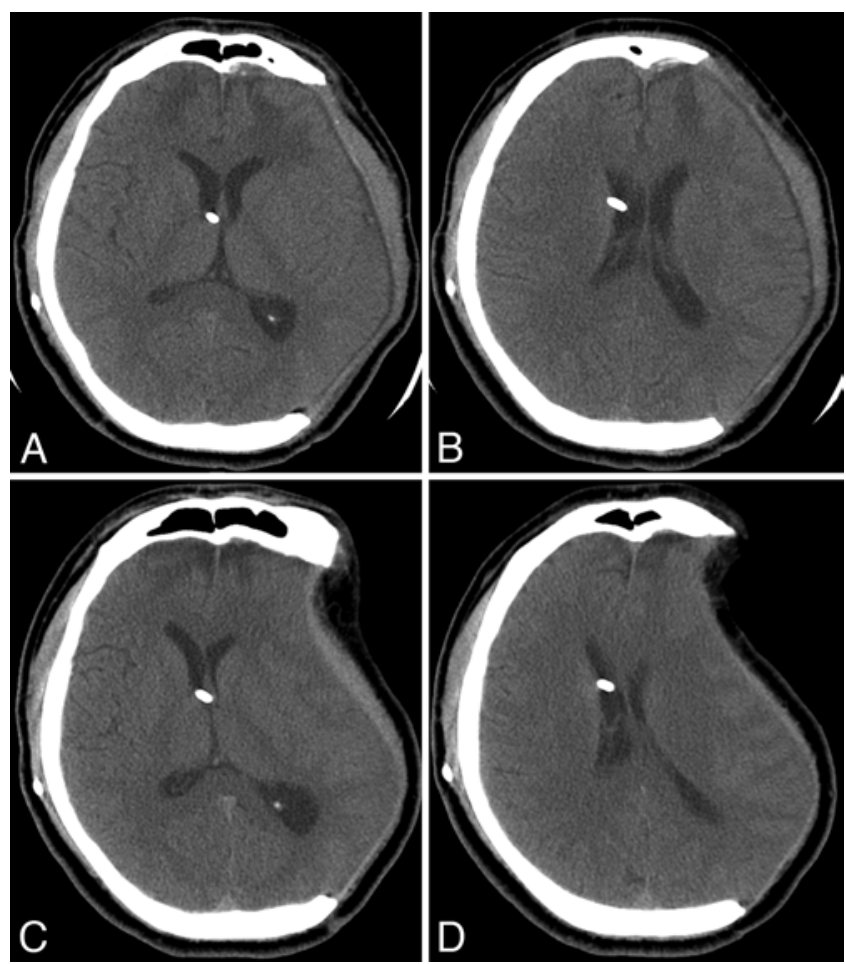

FIG. 2. Patient $B$. Axial head CT images obtained soon after ventriculoperitoneal shunt placement (A and B) and several weeks later ( $C$ and D), showing paradoxical herniation provoked by the shunt.

after ventriculoperitoneal shunting (30\% [exact binomial 95\% CI 6.7\%-65.2\%]).

The number of days between the decompressive craniectomy and the last lumbar puncture, for those who underwent $>1$, was significantly higher for those who deteriorated than for those who did not (mean 206 vs 31 days, respectively; $\mathrm{p}<0.05$ by $\mathrm{t}$-test and the Wilcoxon ranksum test). Most early lumbar punctures were performed to either control intracranial pressure or evaluate fever, whereas most late lumbar punctures were performed to evaluate fever. Only 10 patients had lumbar puncture $>30$ days after the decompressive craniectomy, and 2 of them deteriorated (20\% [exact binomial 95\% CI 2.4\%-55.6\%]). Both of these patients deteriorated within 24 hours of the lumbar puncture.

Brain CT images obtained within 48 hours of the lumbar puncture were available for 9 of these 10 patients; the remaining patient's $\mathrm{CT}$ images were obtained 5 days after the lumbar puncture. These CT scans were reviewed for midline shift, the size of the lateral and third ventricles, and the appearance of the skin flap, which has been characterized as sunken, flat, full, and bulging (see Table 1 in the paper by Yamaura and Makino). ${ }^{22}$ We compared these variables between the 2 patients who deteriorated after the lumbar puncture and the 7 patients who did not. Only 2 variables were significantly different: midline shift and the appearance of the skin flap. The mean midline shift was $0 \mathrm{~mm}$ in the 2 patients who deteriorated and $5.4 \mathrm{~mm}$ in those who did not deteriorate ( 2 -tailed $p<0.05$ by t-test and the Wilcoxon rank-sum test). The shift was toward the side of the decompressive craniectomy except in 1 patient who had a large extraaxial fluid collection on the side of the craniectomy. The skin flap was flat or full before the lumbar puncture in the 2 patients who deteriorated and bulging $(n=6)$ or full $(n=1)$ in those who did not $(p<0.5$ by the chi-square test). None had a sunken appearance.

With respect to shunts, all 10 patients had them placed contralateral to the side of the decompressive craniectomy. The number of days between the decompressive craniectomy and the ventriculoperitoneal shunting and between the shunting and the cranioplasty or death did not differ significantly between those who did and did not deteriorate. In the 3 patients who deteriorated, changes in imaging were variable and occurred as early as 7 days and as late as 62 days after placement of the shunt. At the time the shunt was placed, the craniectomy site was convex with associated hydrocephalus. By the time of deterioration, the site was concave, with the sunken skin flap and paradoxical herniation evident on imaging.

\section{Literature Review}

In 1969, Yamaura and Makino ${ }^{22}$ first observed the syndrome of the sinking skin flap in a patient who spontaneously deteriorated, which prompted them to study 33 patients who underwent decompressive craniectomy for a variety of reasons. To understand better the improvement seen after cranioplasty, they described lumbar punctures performed in a subset of 13 patients immediately before cranioplasty. ${ }^{22}$ With a single exception, the opening pressure was low. No deterioration after the lumbar puncture was observed, but cranioplasty was performed soon thereafter and resulted in improvement in 4 of these 13 patients (31\%). In a subsequent study, 40 patients with craniectomy underwent lumbar puncture with hydrodynamic studies both before and after cranioplasty. ${ }^{3}$ Of these patients, 36 had symptoms, and in 22 of these patients the symptoms were believed to be related to the syndrome of the trephined. The lumbar punctures were performed, on average, 6.5 days before the cranioplasty. The opening pressure was found to be low in all the patients with symptoms and to increase significantly after cranioplasty, but none of the patients was described as deteriorating after the lumbar puncture, which entailed the infusion of artificial cerebrospinal fluid.

In what is likely the first report of provoked paradoxical herniation, deterioration in 2 patients after craniectomy for tumor resection was described and believed to be secondary to an ongoing leak from the lumbar puncture site. ${ }^{5}$ The lumbar punctures had been performed to facilitate tumor resection by lowering elevated intracranial pressure. Deterioration was reversed in these patients with a blood patch. The authors proposed that exposure of the brain to atmospheric pressure allowed for the paradoxical herniation (see Fig. 1 in the paper by Guido and Patterson). ${ }^{5}$ Subsequently, 13 more patients with paradoxical herniation provoked by lumbar puncture were described in a series of 4 cases, whose authors first used the term "paradoxical herniation," ${ }^{\prime \prime 6}$ and in 9 single case reports. ${ }^{2,8-11,14,17,19,20}$

Cases of paradoxical herniation provoked by lumbar puncture are summarized in Table 1. Although the timing was not always easy to determine, lumbar puncture was typically performed $>1$ month after the craniectomy, which had been performed for a variety of conditions, 
TABLE 1. Summary of the literature on patients with deterioration after decompressive craniectomy provoked by lumbar puncture

\begin{tabular}{|c|c|c|c|c|}
\hline Authors \& Year & $\begin{array}{l}\text { Disease Prompting } \\
\text { Decompression }\end{array}$ & $\begin{array}{l}\text { Ventricular } \\
\text { Enlargement }\end{array}$ & $\begin{array}{l}\text { Days to Lumbar } \\
\text { Puncture }\end{array}$ & Comments \\
\hline \multicolumn{5}{|l|}{ Guido \& Patterson, 1976} \\
\hline Case 1 & Meningioma removed & No & Drain at surgery & $\begin{array}{l}\text { Deterioration as patient mobilized after op, be- } \\
\text { lieved to be secondary to lumbar leak }\end{array}$ \\
\hline Case 2 & Metastatic tumor removed & No & Drain at surgery & $\begin{array}{l}\text { Deterioration as patient mobilized after op, be- } \\
\text { lieved to be secondary to lumbar leak }\end{array}$ \\
\hline \multicolumn{5}{|l|}{ Schwab et al., 1998} \\
\hline Case 1 & Cerebral edema & Yes & Unknown & \\
\hline Case 2 & Encephalitis & Yes & 14 & \\
\hline Case 3 & Subdural hematoma & No & Unknown & \\
\hline Case 4 & Ischemic stroke & No & $>30$ & \\
\hline Oyelese et al., 2005 & Ischemic stroke & Yes & $\sim 30$ & \\
\hline Fields et al., 2006 & Trauma & Yes & $\sim 60$ & Already had a ventriculoperitoneal shunt \\
\hline Seinfeld et al., 2007 & Trauma & Yes & 14 & Successfully treated w/ blood patch \\
\hline Vilela, 2008 & Subarachnoid hemorrhage & Yes & 5 & $\begin{array}{l}\text { Deterioration delayed } 43 \text { days because of a pre- } \\
\text { sumed lumbar leak }\end{array}$ \\
\hline Muehlschlegel et al., 2009 & Ischemic stroke & Yes & Unknown & Treated successfully w/ a blood patch \\
\hline Márquez-Romero et al., 2010 & Large spontaneous bleed removed & Yes & 15 & \\
\hline Jadhav \& Venna, 2011 & Brain abscess & Yes & $\sim 120$ & \\
\hline Jung et al., 2012 & Trauma & No & $\sim 35$ & \\
\hline Wang et al., 2014 & Large stroke & No & Unknown & \\
\hline Current series & Trauma & Yes & 54 & \\
\hline Index case & Trauma & Yes & 358 & $\begin{array}{l}\text { See Patient A: Index Case of Deterioration After } \\
\quad \text { Lumbar Puncture }\end{array}$ \\
\hline
\end{tabular}

including encephalitis, abscess, tumor, ischemic stroke, and trauma. The earliest lumbar puncture in a patient with ischemic stroke or trauma was performed 14 days after decompressive craniectomy in a patient with trauma who did not experience severe brain swelling and whose intracranial pressures were normal. ${ }^{17}$ The lumbar puncture at 14 days was performed to address a large subgaleal fluid collection and ventricular enlargement. With one exception, the deterioration provoked by the lumbar puncture was evident within minutes ${ }^{16}$ to 5 days. ${ }^{8}$ In the exceptional case, a lumbar puncture was performed 5 days after decompressive craniectomy for brain swelling related to a subarachnoid hemorrhage. Paradoxical herniation did not occur until 38 days after the lumbar puncture; the cause was presumed to be a lumbar leak. ${ }^{19}$ Most of the cases showed evidence for ventricular enlargement at the time of the lumbar puncture. A recent patient treated with a lumbar puncture followed by a lumbar drain had a bulging skin flap that sank after the drainage of spinal fluid..$^{20}$

Cases of paradoxical herniation provoked by ventriculoperitoneal shunting are summarized in Table 2. In their series of 33 patients, Yamaura and Makino ${ }^{22}$ also described a patient who underwent decompressive craniectomy as part of meningioma resection (Case 18). She developed hydrocephalus and required a shunt 6 weeks after the initial surgery. She subsequently deteriorated slowly but showed marked improvement after cranioplasty performed approximately 1 year after her initial surgery. In another series reported by Schiffer et al., ${ }^{15}$ a patient deteri- orated more immediately after receiving a ventriculoperitoneal shunt and improved after cranioplasty (Case 4). Oh et al. ${ }^{13}$ described deterioration after ventriculoperitoneal shunting in 2 of 13 patients with hydrocephalus who were convex at the craniotomy site before the procedure. "In 2 cases with slit ventricle, one week later, the craniectomy site became excessively sunken, followed by neurological deterioration and aggravated concavity of the scalp flap, especially in an upright position. They improved significantly after changing shunt device (programmable shunt device) and cranioplasty." 13 Two other cases with similar courses were described.6,21 A likely seventh case was described in a review of decompressive craniectomy, although the details were scant (see Fig. 6 in the paper by Akins and Guppy).

\section{Discussion}

Although spontaneous neurological deterioration can occur in patients with decompressive craniectomy, both lumbar puncture and ventriculoperitoneal shunting can provoke deterioration that results from paradoxical herniation. The results of our study of a small series of patients who underwent decompressive craniectomy prompted by trauma suggest that the risk of provoked paradoxical herniation after lumbar puncture may be substantial (20\% [95\% CI 2.4\%-55.6\%]) if > 1 month has passed since the initial injury. Timing may be less important in patients who undergo placement of a ventriculoperitoneal shunt, because the onset of deterioration may be variable 
TABLE 2. Summary of the literature on patients with deterioration after decompressive craniectomy provoked by ventriculoperitoneal shunting

\begin{tabular}{llccc}
\hline Authors \& Year (no. of cases) & $\begin{array}{c}\text { Disease Prompting } \\
\text { Decompression }\end{array}$ & $\begin{array}{c}\text { Enlarged } \\
\text { Ventricles }\end{array}$ & Days to Shunting & Comments \\
\hline Yamaura \& Makino, 1977 & Meningioma removed & Yes & 42 & Case 18 in the series \\
\hline Schiffer et al., 1997 & Meningioma removed & Yes & Unknown & Case 4 in the series \\
\hline Oh et al., 2008 (2) & Acute injury & Yes & Unknown & \\
\hline Acute injury & Yes & Unknown & \\
\hline Han et al., 2008 & Trauma & Yes & 28 & \\
\hline Akins \& Guppy, 2008 & Unknown & Yes & Unknown & Case described in Fig. 6 of the report \\
\hline Wee \& Kuo, 2014 & Trauma & Yes & $\sim 30$ & \\
\hline Current series & Trauma & Yes & 32 & \\
\hline & Trauma & Yes & 42 & \\
\hline Index case & Trauma & Yes & 124 & See Patient B: Index Case of Deterioration After Ventriculo- \\
& Trauma & Yes & 14 & peritoneal Shunting \\
\hline
\end{tabular}

depending on how much fluid is drained. Again, the risk in our small series of patients after shunt placement was substantial (30\% [95\% CI 6.7\%-65.2\%]).

In their seminal work, Yamaura and Makino ${ }^{22}$ described the skin flap in patients after decompressive craniectomy as sunken, flat, full, or bulging. Although the skin flap may be sunken in spontaneous paradoxical herniation, it is typically flat or full, but not bulging, in provoked paradoxical herniation before lumbar puncture. In fact, the patients in most provoked-herniation cases (Table 1 and Fig. 1) showed evidence of ventricular enlargement before lumbar puncture and thus lacked a sunken skin flap. In our series, 7 patients with a bulging skin flap tolerated a lumbar puncture without deterioration, but based on a recent case report, ${ }^{20}$ such an appearance of the skin flap does not afford absolute protection.

The longer the interval between craniectomy and cranioplasty, the greater the risk for spontaneous deterioration, ${ }^{18}$ and the more opportunities potentially exist to provoke deterioration. The increased risk over time may exist especially for patients with trauma. Soon after a trauma that requires decompressive craniectomy, the brain is swollen and the skin flap bulges. In our series, 26 of the 36 patients had undergone lumbar puncture at this stage, and none showed evidence of paradoxical herniation. Conversely, brain swelling decreases over time, and 2 of the remaining 10 patients who had undergone lumbar puncture after 1 month had paradoxical herniation; both of these patients had no midline shift and lacked a bulging skin flap on CT images acquired before the lumbar puncture. In situations other than trauma, the risk may be more immediate, as in the first described patients whose deterioration was believed to be secondary to a spinal fluid leak from a lumbar puncture with a lumbar drain used during brain tumor resection. ${ }^{5}$

Nakamura et al. ${ }^{12}$ may have been the first to suspect a potential risk from ventriculoperitoneal shunting in patients with craniectomy. They described a patient who had a large decompressive craniectomy after trauma. The patient required a ventriculoperitoneal shunt for hydrocephalus and eventually had a cranioplasty. The im- planted plate became infected and had to be removed. The authors noted an immediate neurological deterioration when the patient was upright that could be reversed with the patient lying flat or in the Trendelenburg position. They commented, "The presence of a well-working shunt system is thought to be one of the most important factors in the mechanism which was involved in the development of such marked concave deformity in an erect position."12 Deterioration may occur sooner or later after placement of a ventriculoperitoneal shunt depending on the dynamic between the regression of brain swelling and the amount of fluid drained. For example, in our index case, although the shunt was placed early after the trauma and decompressive craniectomy, deterioration was delayed, presumably reflecting the slow regression of brain swelling. Improvement followed reprograming of the shunt to reduce drainage and a cranioplasty.

The mechanism of paradoxical herniation likely entails exposure of the brain to atmospheric pressure. ${ }^{1,5,15}$ Low rather than high intracranial pressures ensue, which result in sinking of the skin flap and paradoxical herniation. Because the herniation relates to low pressures, treatments aimed at lowering intracranial pressures may worsen the situation. Various treatments for acute deterioration, including intravenous fluids, positioning with the head down, clamping devices that drain cerebrospinal fluid, infusing fluid into the intrathecal space, and performing a blood patch, ${ }^{1}$ have been suggested but are unproven. Regardless of whether deterioration is spontaneous or provoked, improvement can be seen after cranioplasty.

Our study was limited by examining retrospectively at a single institution a single indication for decompressive craniectomy, namely trauma. As a consequence, the numbers are small, and the generalizability is compromised. Nonetheless, most previous investigations have been limited to case reports or small case series. The deterioration provoked by ventriculoperitoneal shunting has not been reported as frequently or with as much detail as that provoked by lumbar puncture. This relative underreporting may be a result of the delay in deterioration that may follow shunting as opposed to the lumbar puncture, as il- 
lustrated by our 2 index cases. More studies are needed to clarify these and other issues related to provoked paradoxical herniation. The ideal study would be a large, prospective, multicenter investigation to examine multiple indications for decompressive craniectomy, which is a study that would pose substantial challenges.

\section{Conclusions}

The risk of provoking paradoxical herniation by lumbar puncture after decompressive craniectomy seems low in the 1st month when the condition that prompted the decompression, such as brain swelling associated with stroke or trauma, will require time to resolve. Although the numbers are small, we found that 1 month after their head trauma, 2 of 10 patients experienced paradoxical herniation provoked by a lumbar puncture. In this setting, lumbar punctures should be avoided if possible, especially if the patient has enlarged ventricles or lacks a bulging skin flap. When the condition that prompted the decompression is addressed with surgery, such as the mass of a brain tumor, the risk of lumbar puncture and lumbar drain provoking paradoxical herniation can be more immediate. ${ }^{5}$ Similarly, patients who undergo decompressive craniectomy and develop hydrocephalus are at risk for developing paradoxical herniation at variable times after the placement of a ventriculoperitoneal shunt, depending on the amount of fluid drained and the stage of brain swelling. In our series, 3 of 10 patients so treated deteriorated. Hence, ventriculoperitoneal shunting should be used cautiously in this setting and special attention devoted to clinical response, amount of fluid drained, and appearance of the skin flap. Cranioplasty eliminates the risk of paradoxical herniation and should be considered sooner rather than later in such patients.

\section{Acknowledgment}

We appreciate the efforts of Rebecca Masters in screening medical records to identify patients who underwent lumbar puncture or ventriculoperitoneal shunting.

\section{References}

1. Akins PT, Guppy KH: Sinking skin flaps, paradoxical herniation, and external brain tamponade: a review of decompressive craniectomy management. Neurocrit Care 9:269-276, 2008

2. Fields JD, Lansberg MG, Skirboll SL, Kurien PA, Wijman CA: "Paradoxical" transtentorial herniation due to CSF drainage in the presence of a hemicraniectomy. Neurology 67:1513-1514, 2006

3. Fodstad H, Love JA, Ekstedt J, Fridén H, Liliequist B: Effect of cranioplasty on cerebrospinal fluid hydrodynamics in patients with the syndrome of the trephined. Acta Neurochir (Wien) 70:21-30, 1984

4. Grant FC, Norcross NC: Repair of cranial defects by cranioplasty. Ann Surg 110:488-512, 1939

5. Guido LJ, Patterson RH Jr: Focal neurological deficits secondary to intraoperative CSF drainage: successful resolution with an epidural blood patch. Report of two cases. J Neurosurg 45:348-351, 1976

6. Han PY, Kim JH, Kang HI, Kim JS: "Syndrome of the sinking skin-flap" secondary to the ventriculoperitoneal shunt after craniectomy. J Korean Neurosurg Soc 43:51-53, 2008

7. Honeybul S, Janzen C, Kruger K, Ho KM: The impact of cranioplasty on neurological function. Br J Neurosurg 27:636-641, 2013

8. Jadhav AP, Venna N: Teaching NeuroImages: Gravity reverses paradoxical herniation in the sinking brain syndrome. Neurology 77:e42, 2011

9. Jung HJ, Kim DM, Kim SW: Paradoxical transtentorial herniation caused by lumbar puncture after decompressive craniectomy. J Korean Neurosurg Soc 51:102-104, 2012

10. Márquez-Romero JM, Zermeño-Pohls F, Soto-Cabrera E: [Paradoxical herniation due to a continuous cerebrospinal fluid drain in a previously craniectomised patient.] Neurologia 25:269-271, 2010 (Span)

11. Muehlschlegel S, Voetsch B, Sorond FA: Emergent epidural blood patch: lifesaving treatment of paradoxical herniation. Arch Neurol 66:670-671, 2009

12. Nakamura T, Takashima T, Isobe K, Yamaura A: Rapid neurological alteration associated with concave deformity of the skin flap in a craniectomized patient. Case report. Neurol Med Chir (Tokyo) 20:89-93, 1980

13. Oh CH, Park CO, Hyun DK, Park HC, Yoon SH: Comparative study of outcomes between shunting after cranioplasty and in cranioplasty after shunting in large concave flaccid cranial defect with hydrocephalus. J Korean Neurosurg Soc 44:211-216, 2008

14. Oyelese AA, Steinberg GK, Huhn SL, Wijman CA: Paradoxical cerebral herniation secondary to lumbar puncture after decompressive craniectomy for a large space-occupying hemispheric stroke: case report. Neurosurgery 57:E594, 2005

15. Schiffer J, Gur R, Nisim U, Pollak L: Symptomatic patients after craniectomy. Surg Neurol 47:231-237, 1997

16. Schwab S, Erbguth F, Aschoff A, Orberk E, Spranger M, Hacke W: ["Paradoxical" herniation after decompressive trephining.] Nervenarzt 69:896-900, 1998 (Ger)

17. Seinfeld J, Sawyer M, Rabb CH: Successful treatment of paradoxical cerebral herniation by lumbar epidural blood patch placement: technical case report. Neurosurgery 61 (3 Suppl):E175, 2007

18. Stiver SI, Wintermark M, Manley GT: Reversible monoparesis following decompressive hemicraniectomy for traumatic brain injury. J Neurosurg 109:245-254, 2008

19. Vilela MD: Delayed paradoxical herniation after a decompressive craniectomy: case report. Surg Neurol 69:293-296, 2008

20. Wang QP, Zhou ZM, You C: Paradoxical herniation caused by cerebrospinal fluid drainage after decompressive craniectomy. Neurol India 62:79-80, 2014

21. Wee HY, Kuo JR: Never neglect the atmospheric pressure effect on a brain with a skull defect. Int Med Case Rep J 7:67-69, 2014

22. Yamaura A, Makino H: Neurological deficits in the presence of the sinking skin flap following decompressive craniectomy. Neurol Med Chir (Tokyo) 17:43-53, 1977

\section{Author Contributions}

Conception and design: all authors. Acquisition of data: Creutzfeldt, Longstreth. Analysis and interpretation of data: Creutzfeldt, Longstreth. Drafting the article: all authors. Critically revising the article: all authors. Reviewed submitted version of manuscript: all authors. Approved the final version of the manuscript on behalf of all authors: Creutzfeldt. Statistical analysis: Longstreth. Administrative/technical/material support: Longstreth. Study supervision: Vilela, Longstreth.

\section{Correspondence}

Claire Creutzfeldt, Harborview Medical Center, Department of Neurology, 325 Ninth Ave., Box 359775, Seattle, WA 981042420.email: clairejc@uw.edu. 